\title{
A Structural Mechanics Approach for the Vibrational Analysis of Single-Layered Graphene Sheets
}

\author{
Andika Putra, Mikrajuddin Abdullah, and Khairurrijal \\ Physics of Electronic Materials Research Division, \\ Faculty of Mathematics and Natural Sciences, \\ Institut Teknologi Bandung \\ e-mail address: krijal@fi.itb.ac.id
}

\begin{abstract}
A structural mechanics approach has been employed to analyze the vibrational behavior of single-layered graphene sheets. By adopting this approach, natural frequencies and mode shapes are obtained for different chirality and imposed boundary conditions. Numerical results from the implemented modeling are examined to provide predictive equations for fundamental frequencies of cantilevered and bridged graphene sheets. With the proposed equations, the natural frequencies can be predicted and are comparable to those obtained from theoretical consideration and experimental results.
\end{abstract}

Keywords: Single-layered graphene sheets, Structural mechanics, Vibrational analysis

\section{Introduction}

The advancement of science and technology has evolved into the era of nanotechnology. Among many nanostructure materials, graphene is the most popular topic in physics today. Individual graphene sheets, which are monolayers of carbon atoms arranged in a honeycomb network and become the thinnest objects imaginable, have for the first time been isolated by peeling them off from graphite with Scotch tape ${ }^{1)}$. A general and reproducible approach in preparing graphene sheets from graphite has been found by Stankovich et $a l^{2)}$. Future directions of research and applications of graphene sheets have also been reviewed ${ }^{3)}$.

Because of their lightness and stiffness, nanoelectromechanical resonators have been fabricated from graphene sheets ${ }^{4)}$. Studies on mechanical properties of graphene sheets are therefore important for developing various nanoelectromechanical systems. Many theoretical studies on mechanical properties of graphene sheets have been started from those of carbon nanotubes. $\mathrm{Li}$ and $\mathrm{Chou}^{5}$ proposed an atomistic modeling, which is based on structural mechanics, to study vibrational behavior of single- and multi-walled-carbon-nanotube (SWCNT and MWCNT)-based nanomechanical resonators $^{6,7)}$ and their applications for mass detection, strain and pressure sensing ${ }^{8,9)}$. Tserpes and Papanikos ${ }^{10)}$ further proposed finite element modeling of SWCNTs with varied thickness, similar to $\mathrm{Li}$ and Chou's model. Based on the models proposed above, Sakhaee-Pour et al. then studied the vibrational behavior of single-layered graphene sheets $(\mathrm{SLGSs})^{11)}$, its potential application as strain sensor ${ }^{12}$ ) and mass sensors or atomistic dust detectors ${ }^{13)}$.

In the models that have been suggested above, computational processes needed in determining the mechanical properties commonly employ commercial finite element softwares. In the finite element modeling, memory storage and computer processing time may become obstacles. Thus, the challenge in present work of vibrational analysis of SLGSs is to perform the simulations on a low cost home desktop PC (using $2.40 \mathrm{GHz}$ Intel Pentium $4 \mathrm{CPU}$ and only $512 \mathrm{MB}$ of DDR RAM). Although the simulations that can be done are limited, the results obtained are still reliable as compared to the experimental results.

\section{Molecular Structural Mechanics Applied to SLGS}

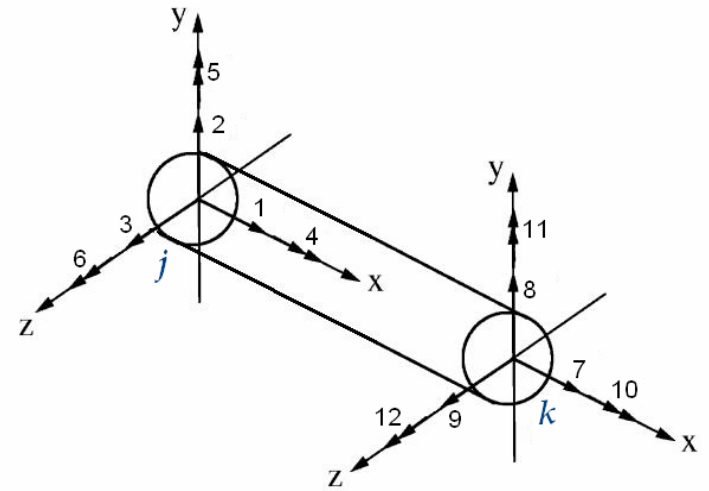

Figure 1. Illustration of a beam element in a space frame.

For a beam element with uniform circular cross section in a space frame (Figure 1), the elemental equilibrium equation in the local coordinate system can be written as ${ }^{14)}$

$$
\mathbf{K}^{\mathbf{e}} \mathbf{u}^{\mathbf{e}}=\mathbf{f}^{\mathbf{e}},
$$

where

$\mathbf{u}^{\mathbf{e}}=\left[u_{x j}, u_{y j}, u_{z j}, \phi_{x y}, \phi_{y j}, \phi_{z j}, u_{x k}, u_{y k}, u_{z k}, \phi_{x k}, \phi_{y k}, \phi_{z k}\right]^{\mathbf{T}}$, 
$\mathbf{f}^{\mathbf{e}}=\left[f_{x j}, f_{y j}, f_{z j}, m_{x y}, m_{y j}, m_{z j}, f_{x k}, f_{y k}, f_{z k}, m_{x k}, m_{y k}, m_{z k}\right]^{\mathrm{T}}$,

are the nodal displacement vector and nodal force vector of the element, respectively. $\mathbf{K}^{\mathbf{e}}$ is the elemental stiffness matrix, which consists of the following submatrices

$$
\begin{aligned}
& \mathbf{K}^{\mathbf{e}}=\left[\begin{array}{ll}
\mathbf{K}_{\mathbf{j j}} & \mathbf{K}_{\mathbf{j k}} \\
\mathbf{K}_{\mathbf{j k}}^{\mathbf{T}} & \mathbf{K}_{\mathbf{k k}}
\end{array}\right], \\
& \mathbf{K}_{\mathbf{j j}}=\left[\begin{array}{cccccc}
a & 0 & 0 & 0 & 0 & 0 \\
0 & 6 b / L & 0 & 0 & 0 & 3 b \\
0 & 0 & 6 b / L & 0 & -3 b & 0 \\
0 & 0 & 0 & c & 0 & 0 \\
0 & 0 & -3 b & 0 & 2 b L & 0 \\
0 & 3 b & 0 & 0 & 0 & 2 b L
\end{array}\right] \text {, } \\
& \mathbf{K}_{\mathbf{j k}}=\left[\begin{array}{cccccc}
-a & 0 & 0 & 0 & 0 & 0 \\
0 & -6 b / L & 0 & 0 & 0 & 3 b \\
0 & 0 & -6 b / L & 0 & -3 b & 0 \\
0 & 0 & 0 & -c & 0 & 0 \\
0 & 0 & 3 b & 0 & b L & 0 \\
0 & -3 b & 0 & 0 & 0 & b L
\end{array}\right] \text {, } \\
& \mathbf{K}_{\mathbf{k k}}=\left[\begin{array}{cccccc}
a & 0 & 0 & 0 & 0 & 0 \\
0 & 6 b / L & 0 & 0 & 0 & -3 b \\
0 & 0 & 6 b / L & 0 & 3 b & 0 \\
0 & 0 & 0 & c & 0 & 0 \\
0 & 0 & 3 b & 0 & 2 b L & 0 \\
0 & -3 b & 0 & 0 & 0 & 2 b L
\end{array}\right],
\end{aligned}
$$

in which $a=E A / L, b=2 E I / L^{2}, c=G J / L$, while $E$ is the Young's modulus and $G$ is the shear modulus. The beam cross section diameter $d$ is related to the beam properties as

$$
A=\frac{\pi d^{2}}{4}, \quad I=\frac{\pi d^{4}}{64}, \quad J=\frac{\pi d^{4}}{32},
$$

where $A$ is the cross section area, $I$ is the moment of inertia, and $J$ is the polar moment of inertia.

In classical structural mechanics, the strain energy of a uniform circular beam under axial force $N$, under pure bending moment $M$ and under pure torsion $T$, respectively, are

$$
\begin{aligned}
& U_{A}=\frac{1}{2} \int_{0}^{L} \frac{N^{2}}{E A} d L=\frac{1}{2} \frac{N^{2} L}{E A}=\frac{1}{2} \frac{E A}{L}(\Delta L)^{2}, \\
& U_{M}=\frac{1}{2} \int_{0}^{L} \frac{M^{2}}{E I} d L=\frac{2 E I}{L} \alpha^{2}=\frac{1}{2} \frac{E I}{L}(2 \alpha)^{2}, \\
& U_{T}=\frac{1}{2} \int_{0}^{L} \frac{T^{2}}{G J} d L=\frac{1}{2} \frac{T^{2} L}{G J}=\frac{1}{2} \frac{G J}{L}(\Delta \beta)^{2},
\end{aligned}
$$

where $\Delta L$ is the axial stretching deformation, $\alpha$ is the rotational angle at the ends of the beam, and $\Delta \beta$ is the relative rotation between the ends of the beam.

In molecular structural mechanics approach, a single-layered graphene sheet or carbon nanotube is modeled as a space frame structure, with the covalent bonds regarded as connecting beams and carbon atoms as joint nodes. $\mathrm{Li}$ and $\mathrm{Chou}^{5}$ ) have established the energy equivalence between total steric potential energy in the microscopic computational chemistry and the macroscopic structural mechanics. The total steric potential energy (omitting the electrostatic interaction) is a sum of energies due to bonded interactions and non-bonded interactions. By neglecting van der Waals forces and imposing small deformations to the system (adopting harmonic approximation in describing the energy), the total potential energy for the system under consideration is

$$
U=\sum U_{r}+\sum U_{\theta}+\sum U_{\tau},
$$

where $U_{r}$ is the bond stretching energy, which is expressed as

$$
U_{r}=\frac{1}{2} k_{r}\left(r-r_{0}\right)^{2}=\frac{1}{2} k_{r}(\Delta r)^{2},
$$

$U_{\theta}$ is the angle bending energy,

$$
U_{\theta}=\frac{1}{2} k_{\theta}\left(\theta-\theta_{0}\right)^{2}=\frac{1}{2} k_{\theta}(\Delta \theta)^{2},
$$

$U_{\tau}$ is the torsional potential energy, the sum of out-ofplane and dihedral angle torsional energies, and can be obtained as

$$
U_{\tau}=U_{\phi}+U_{\omega}=\frac{1}{2} k_{\tau}(\Delta \phi)^{2},
$$

where $k_{r}, k_{\theta}$ and $k_{\tau}$ are the bond stretching force constant, bond angle bending force constant and torsional resistance respectively, and the symbols $\Delta r$, $\Delta \theta$ and $\Delta \phi$ represent the corresponding displacements from the equilibrium positions.

Since distinct types of stored energies are independent in both molecular and structural mechanics, the equivalency between force constants and stiffness parameters are then deduced as

$$
\frac{E A}{L}=k_{r}, \quad \frac{E I}{L}=k_{\theta}, \quad \frac{G J}{L}=k_{\tau} .
$$

From Eq. (8) and (16), properties of the equivalent structural beam element can be obtained as ${ }^{10)}$

$$
d=4 \sqrt{\frac{k_{\theta}}{k_{r}}}, \quad E=\frac{k_{r}^{2} L}{4 \pi k_{\theta}^{2}}, \quad G=\frac{k_{r}^{2} k_{\tau} L}{8 \pi k_{\theta}^{2}},
$$

In order to analyze the vibration of SLGSs, each carbon-carbon covalent bond is considered as a circular beam. The equations of motion are imposed for each element in local coordinates and transformed with respect to the global coordinate system. Each separate beam element is then assembled into a single set of equations. By imposing the equation of motion 
for free undamped harmonic vibration, we obtain the vibrational equation of motion for SLGSs as

$$
\begin{aligned}
& {[M]\{\ddot{u}\}+[K]\{u\}=0,} \\
& {[K]\{u\}=\omega^{2}[M]\{u\},} \\
& {[K]\{u\}=\lambda[M]\{u\},}
\end{aligned}
$$

where $[K]$ is the overall stiffness matrix, $[M]$ is the overall mass matrix, and $\omega=2 \pi f$ is the angular vibrational frequency of the structures. The resonant frequencies and corresponding mode shapes can be obtained by solving the eigenvalue problem above.

Each carbon nuclei $\left(m_{c}=1.9943 \times 10^{26} \mathrm{~kg}\right)$ is assumed to be spherical and have concentrated mass, since its small radius $\left(r_{c}=2.75 \times 10^{-5} \AA\right)$ compared to the length of the $\mathrm{C}-\mathrm{C}$ bonds ( $L=1.421 \AA$ ). The carbon atom lies on joint nodes of the beams. Consequently, $[M]$ will be sparse and symmetrical with only real and non-zero (positive) entries in all the diagonal components. Such matrix is a positive definite matrix which yields different real positive eigenvalues and real eigenvectors ${ }^{15)}$. Because each node has three neighboring nodes at maximum, $[K]$ is also a sparse symmetrical matrix.

Such problem is common in structural mechanics. A common procedure for solving such real symmetric generalized eigenvalue problems is using Lanczos algorithms ${ }^{16)}$, which are highly effective in solving large symmetric eigenvalue computations, where the matrices are sparse.

Because the components of the overall mass matrix due to rotational displacements $\left(\frac{2}{3} m_{c} r_{c}^{2}\right)$ are extremely small compared to the components of mass due to translational displacements $\left(m_{c}\right)$, static condensation method may be used to reduce the size of the $[K]$ and $[M]$ matrices $^{6,12}$ ). In the static condensation method, the initial $[K]$ matrix can be reduced till a quarter of its initial dimension and $[M]$ matrix does not need to be assembled. Although this yields smaller dimension of condensed matrix $[K]_{s}$, the matrix $[K]_{s}$, is not sparse. This condition prolongs the computational time and it actually increases the memory storage usage. Therefore, Lanczos procedures are preferable in present work.

\section{Different Boundary Conditions of the SLGSs}

Several boundary conditions are then applied to the SLGS model since chirality ${ }^{17)}$ has a significant role on the mechanical properties of graphene. The zigzag and armchair ${ }^{17}$ models of single-layered graphene sheet are simulated in this study. The zigzag and armchair models of SLGSs under consideration are (Figure 2)

1. cantilevered zigzag sheet clamped on one edge,

2. cantilevered armchair sheet clamped on one edge,

3. bridged zigzag sheet clamped on two edges,

4. bridged armchair zigzag sheet clamped on two edges,

5. sheet clamped on four edges.
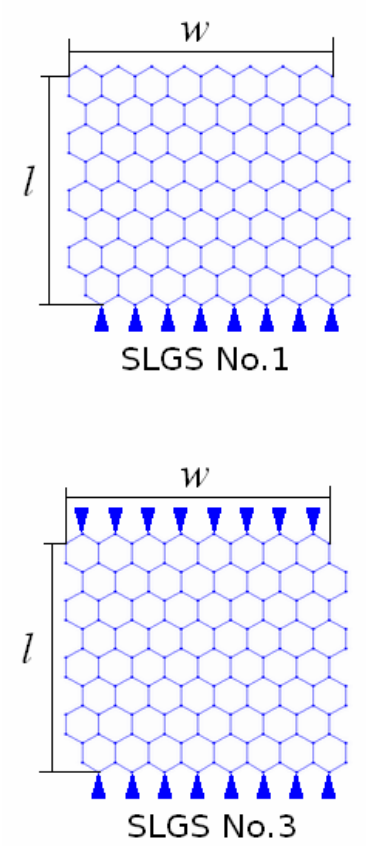
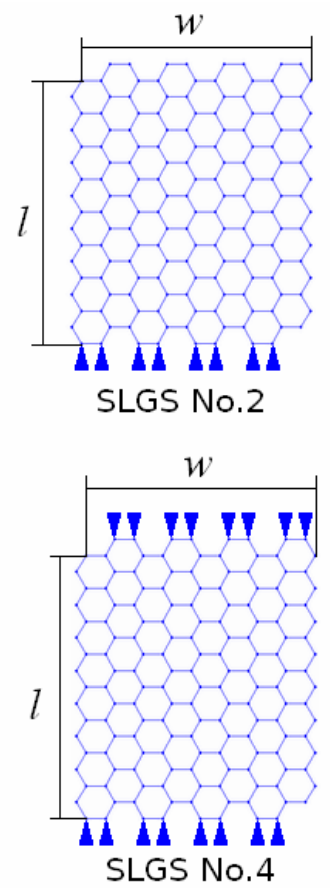

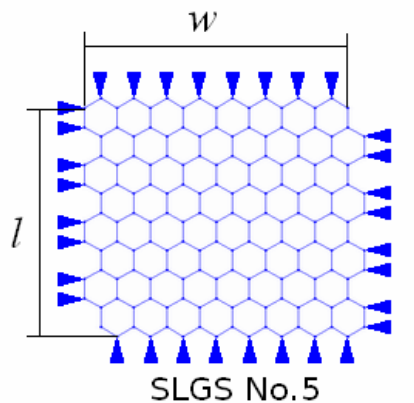

SLGS No. 5

Figure 2. Different boundary conditions of the single-layered graphene sheets. 


\section{Results and Discussion}

The vibrational properties of considered SLGSs are investigated. The force field constants of the covalent bonds between carbon-carbon atoms are chosen to be ${ }^{11)} k_{r}=6.52 \times 10^{-7} \mathrm{~N} / \mathrm{nm}, k_{\theta}=8.76 \times 10^{-10}$ $\mathrm{N} \mathrm{nm} / \mathrm{rad}^{2}$, and $k_{\tau}=2.78 \times 10^{-10} \mathrm{~N} \mathrm{~nm} / \mathrm{rad}^{2}$. The length of the beam element is $L=1.421 \AA$ and from Eq. (16), the elemental beam properties can be calculated. The diameter of the beam obtained, which is taken to be the thickness of graphene sheet, from Eq. (17) is $t=d$ $=0.147 \mathrm{~nm}$. The numerical values used in the eigenvalue computation of Eq. (20) for analyzing the vibrational behavior of SLGS can be summarized as in Table 1.

Table 1: Numerical values for the parameters used in the simulation (units are chosen as $\mathrm{kg}, \mathrm{nm}$, and s)

\begin{tabular}{|l|l|l|}
\hline \multicolumn{1}{|c|}{ Parameters } & \multicolumn{1}{|c|}{ Description } & \multicolumn{1}{c|}{ Numerical values } \\
\hline \hline$L$ & $\begin{array}{l}\text { length of beam } \\
\text { element }\end{array}$ & $0.1421 \mathrm{~nm}$ \\
\hline$a=E A / L$ & & $652 \mathrm{~kg} / \mathrm{s}^{2}$ \\
\hline$B=2 E I / L^{2}$ & & $12.33 \mathrm{~kg} \mathrm{~nm} / \mathrm{s}^{2} \mathrm{rad}^{2}$ \\
\hline$c=G J / L$ & & $0.278 \mathrm{~kg} \mathrm{~nm} / \mathrm{s}^{2} \mathrm{rad}^{2}$ \\
\hline$t=d$ & thickness of & $0.147 \mathrm{~nm}$ \\
\hline$m_{c}$ & $\begin{array}{l}\text { mass of carbon } \\
\text { atom }\end{array}$ & $1.9943 \times 10^{-26} \mathrm{~kg}$ \\
\hline$\frac{2}{3} m_{c} r_{c}^{2}$ & rotational mass & $5.042 \times 1.9943 \times 10^{-36} \mathrm{~kg} \mathrm{~nm}^{2}$ \\
\hline
\end{tabular}

Fundamental frequencies of SLGS No.1-4 versus side length $l$ are shown in Figures 3 and 4, at which the results computed are limited to $l<55 \mathrm{~nm}$. The first resonant frequencies for SLGS nos. 1-4 are calculated for different aspect ratios and results show that they do not depend on the chirality and aspect ratio. Instead, the frequencies decrease nonlinearly with the side length $l$.

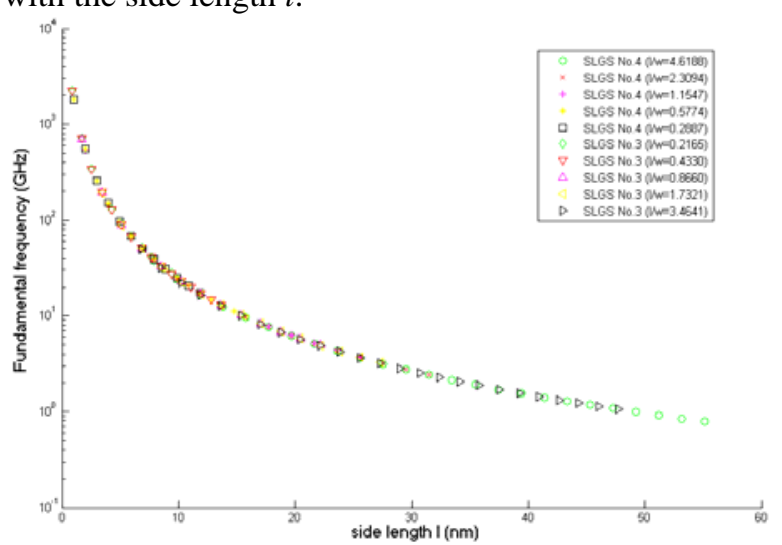

Figure 3. Fundamental frequencies of cantilevered SLGS with one clamped edge and different aspect ratios.

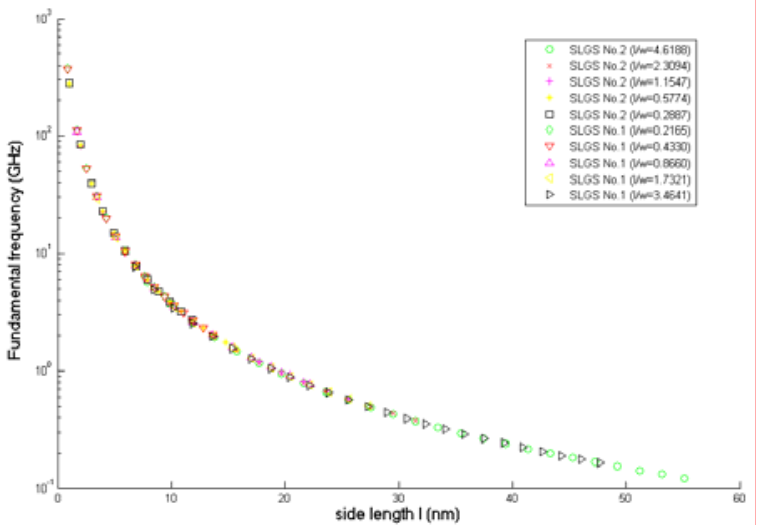

Figure 4. Fundamental frequencies of bridged SLGS with two clamped edges and different aspect ratios.

The mode shapes of the SLGSs are obtained from the computed eigenvectors of Eq. (20). The first eight mode shapes of sheets nos. 1, 3, and 5 simulated are displayed in Figure 5-7. The obtained mode shapes are very similar with those of Sakhaee-Pour et al's work $^{11)}$. The first four mode shapes are exactly the same compared to the results presented on Ref. ${ }^{8}$, although different parameter for the SLGS thickness is used (in present work the thickness of SLGS is selected to be $t=0.147 \mathrm{~nm}$ while in Ref. ${ }^{11)}$ it is $t=0.34 \mathrm{~nm})$.

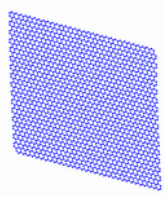

(1)

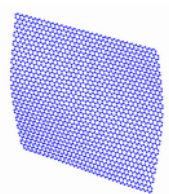

(5)

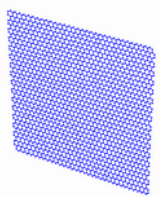

(2)

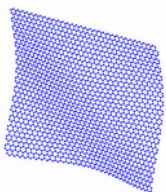

(6)

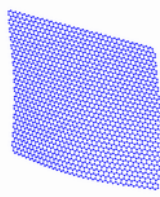

(3)

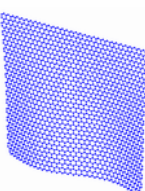

(7)

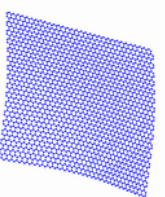

(4)

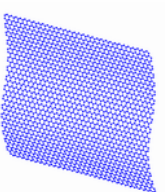

(8)
Figure 5. First eight mode shapes of SLGS No.1 for $l=6.8208 \mathrm{~nm}$ and $l / w=0.8660$.

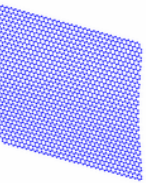

(1)

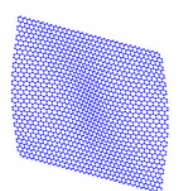

(5)

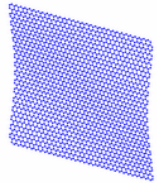

(2)

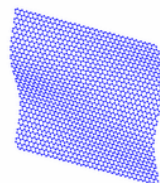

(6)

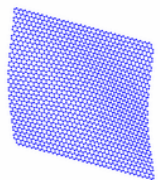

(3)

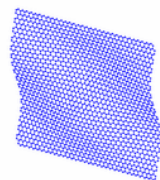

(7)

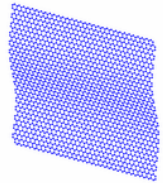

(4)

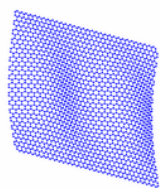

(8)
Figure 6. First eight mode shapes of SLGS No.3 for $l=6.8208 \mathrm{~nm}$ and $l / w=0.8660$. 


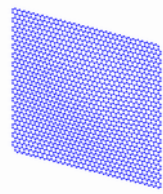

(1)

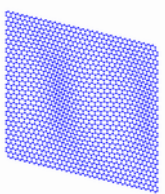

(5)

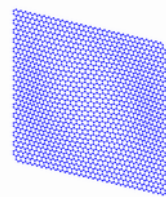

(2)

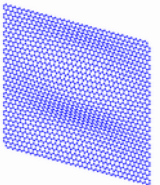

(6)

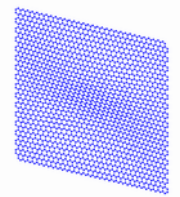

(3)

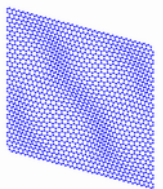

(7)

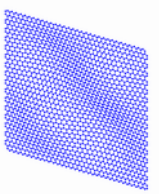

(4)

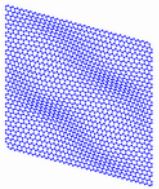

(8)
Figure 7. First eight mode shapes of SLGS No.5 for $l=6.8208 \mathrm{~nm}$ and $l / w=0.8660$.

Ultrahigh frequency nanomechanical resonators can be achieved using SLGSs. The fundamental frequencies of SLGSs with lengths in the range from $155 \mathrm{~nm}$ and length over width ratios of 0.22-4.62 are calculated as displayed in Figures 3 and 4. For both imposed boundary conditions, the fundamental frequency increases with the reduction in SLGS side length, in which chirality does not have a significant effect on the fundamental frequency. The fact that the bridge and cantilever boundary conditions have sinusoidal and/or hyperbolic sine and cosine configurations in the first mode (as can be seen from Figures 5 and 6) yields fundamental frequencies that are independent to the aspect ratio.

The fundamental frequencies obtained are in the ranges of $0.1-280 \mathrm{GHz}$ and $0.8-2000 \mathrm{GHz}$, respectively, for cantilevered and bridged SLGSs. Therefore, the resonator devices designed on the basis of the SLGSs are capable of measuring activated frequencies with high and broad ranges of sensitivities.

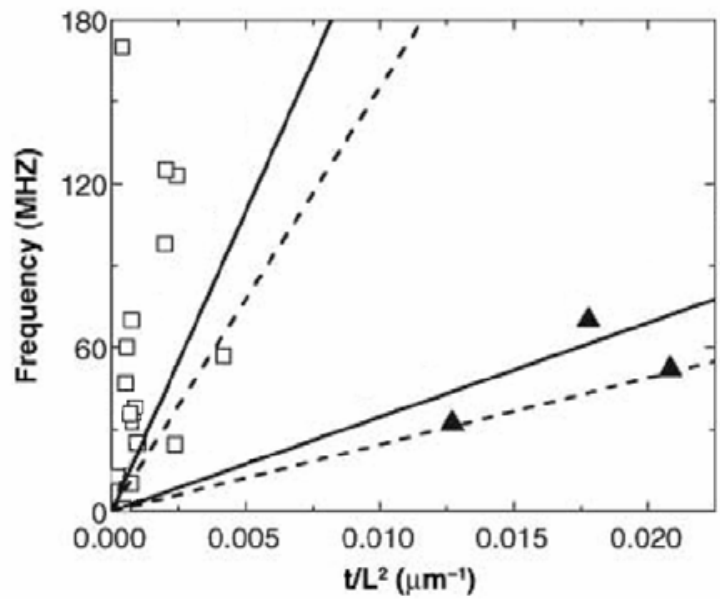

Figure 8. The fundamental mode of all the doubly clamped sheets and cantilevers versus $t / l^{2}$. Cantilevers, triangles; doubly clamped sheets with $t<7 \mathrm{~nm}$, open squares. The dashed lines correspond to Eq. (22) with $E=0.5 \mathrm{TPa}$.
The computed results for fundamental frequencies are then compared to experimental observations done by Bunch et al. ${ }^{4)}$. A plot showing the frequency of the fundamental mode of all the doubly clamped (bridged) sheets and cantilevers versus $t / l^{2}$ observed by Bunch et al. is presented in Figure 8. In their work, the experimental results are compared to the theoretical prediction for the fundamental resonance mode $f_{0}$ for mechanical resonators under tension $T$, which is given by ${ }^{18)}$

$$
f_{0}=\left\{\left[A(E / \rho)^{1 / 2} t / l^{2}\right]^{2}+A^{2} 0.57 T / \rho l^{2} w t\right\}^{2},
$$

where $E$ is the Young's modulus; $\rho$ is the mass density; $t, w$, and $l$ are the dimensions of the suspended graphene sheet (thickness, width, and side length, respectively); and the clamping coefficient, $A$, is 1.03 for bridged sheets and 0.162 for cantilevers. In the limit of small tension, Eq. (21) becomes

$$
f_{0}=A(E / \rho)^{1 / 2} t / l^{2},
$$

where the resonant frequency $f_{0}$ scales to $t / l^{2}$.

Bunch et $a l .{ }^{4)}$ show in their work that the fundamental frequency follows the prediction in Eq. (22) reasonably, which corresponds to the value of $E=$ $0.5 \mathrm{TPa}$ and $\rho=2200 \mathrm{~kg} / \mathrm{m}^{3}$ (known values for bulk graphite mass density), as can be seen in Figure 8. The resonant frequencies for the doubly clamped beams with $t<7 \mathrm{~nm}$ show more scatter, which is likely due to the tension results from the fabrication process.

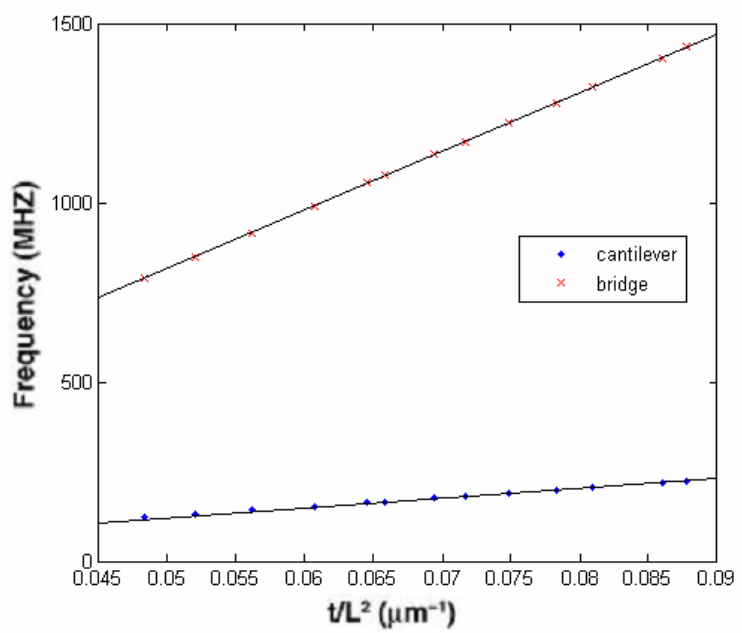

Figure 9. Fundamental frequencies of SLGS No.1-4 as a function of $t / l^{2}$ with side length $l>40 \mathrm{~nm}$.

Present computed results for fundamental frequencies of SLGS nos. 1-4 with the side length $l>$ $40 \mathrm{~nm}$ are scaled to $t / l^{2}$ (Figure 9). The predictive equation, using least squares regression, for cantilevers (SLGS nos. 1-2) is

$$
f_{0}=2529 t / l^{2},
$$

where $f_{0}$ in $\mathrm{MHz}$ and $t / \mathrm{l}^{2}$ in $\mu \mathrm{m}^{-1}$, and for bridge sheets (SLGS No.3-4) is 


$$
f_{0}=16323 t / l^{2}+1
$$

The correlation coefficient $R^{2}$ is found to be greater than 0.99 for both considered data, which demonstrates a good linear behavior of the fundamental frequency $f_{0}$ as a function of $t / l^{2}$. By substituting the parameters $E=0.5 \mathrm{TPa}$ and $\rho=2200$ $\mathrm{kg} / \mathrm{m}^{3}$ in Eq. (22), the gradients obtained for the resonant frequency $f_{0}$ scales to $t / l^{2}$ are $2442 \mathrm{MHz} \mu \mathrm{m}$ and $15528 \mathrm{MHz} \mu \mathrm{m}$, for cantilevered and doubly clamped sheets, respectively. Hence, the predictive equations for the computed fundamental frequencies in present work show good agreement with theoretical considerations in Eq. (22), whereas the gradients are within 5\% difference. Equations (23) and (24)) provide good predictive models to determine the fundamental frequencies of cantilevered and bridged SLGSs, which can be used to design desirable nanomechanical resonators, consequently.

\section{Conclusions}

A structural mechanics approach for the vibrational analysis of single-layered graphene sheets (SLGSs) has been performed. Several boundary conditions on natural frequencies and mode shapes were investigated. The fundamental frequencies of cantilevered and bridged SLGSs were calculated and results showed that chirality and aspect ratio do not have impact on the fundamental frequencies. The ultrahigh resonant frequencies of SLGSs provide promising applications of SLGSs as nanomechanical resonators. It is predicted that SLGSs have the advantage of producing sensitive resonators.

On the basis of the modeling in present work, equations for cantilevered and bridged graphene sheets natural frequency prediction have been established. These equations fit the theoretical consideration well and show good agreement between the molecular structural mechanics prediction and the experimental investigation of SLGSs-based nanomechanical resonator. Therefore, these allow the design of desirable ultrahigh nanomechanical resonators.

\section{References}

1. K. S. Novoselov et al., Electric field effect in atomically thin carbon films, Science, 306, 666669, 2004.

2. S. Stankovich et al., Synthesis of graphene-based nanosheets via chemical reduction of exfoliated graphite oxide, Carbon, 45, 1558-1565, 2007.

3. A. K. Geim, Graphene: status and prospects, Science, 324, 1530-1534, 2009.
4. J. S. Bunch et al., Electromechanical resonators from graphene sheets, Science, 315, 490-493, 2008.

5. C. Li, T-W. Chou, A structural mechanics approach for the analysis of carbon nanotubes, Int J Solids Struct, 40, 2487-2499, 2003.

6. C. Li, T-W. Chou, Single-walled carbon nanotubes as ultrahigh frequency nanomechanical resonators, Phys. Rev. B, 68:1, 073405, 2003.

7. C. Li and T-W. Chou, Vibrational behaviors of multiwalled-carbon-nanotube-based nanomechanical resonators, Appl. Phys. Lett., 84:1, 121-123, 2004.

8. C. Li and T-W. Chou, Mass detection using carbon nanotube-based nanomechanical resonators, Appl. Phys. Lett., 84:25, 5246-5248, 2004.

9. C. Li and T-W. Chou, Strain and pressure sensing using single-walled carbon nanotubes, Nanotechnology, 15, 1493-1496, 2004.

10. K. Tserpes and P. Papanikos, Finite element modeling of single-walled carbon nanotubes, Composite: Part B 36, 468-477, 2005.

11. A. Sakhaee-Pour, M. T. Ahmadian, and R. Naghdabadi, Vibrational analysis of singlelayered graphene sheets, Nanotechnology, 19, 085702, 2008.

12. A. Sakhaee-Pour, M. T. Ahmadian, and A. Vafai, Potential application of single-layered graphene sheet as strain sensor, Solid State Commun., 147, 336-340, 2008.

13. A. Sakhaee-Pour, M. T. Ahmadian, and A. Vafai, Applications of single-layered graphene sheets as mass sensors and atomistic dust detectors, Solid State Commun., 145, 168-172, 2008.

14. W. Weaver Jr. and J. M. Gere. Matrix Analysis of Framed Structures, 2 ed. D. Van Nostrand Company, New York, 1980.

15. D. S. Watkins, Fundamentals of Matrix Computations, 2 ed. Pure and Applied Mathematics. John Wiley \& Sons, Inc., New York, 2002.

16. J. K. Cullum and R. A. Willoughby, Lanczos Algorithms for Large Symmetric Eigenvalue Computations, vol. 1 of Classics in Applied Mathematics, Society for Industrial and Applied Mathematics, Philadelphia, 2002.

17. R. Saito, G. Dresselhaus, and M. S. Dresselhaus, Physical Properties of Carbon Nanotubes, Imperial College Press, London, ch. 4, 35-58, 1998.

18. S. Timoshenko, D. H. Young, and W. Weaver, Vibration Problems in Engineering, 4 ed. Wiley, New York, 425-427, 1974. 